\title{
A Neural Lyapunov Approach to Transient Stability Assessment in Interconnected Microgrids
}

\author{
Tong Huang \\ Texas A\&M University \\ tonghuang@tamu.edu
}

\author{
Sicun Gao \\ University of California, \\ San Diego \\ sicung@ucsd.edu
}

\author{
Xun Long \\ Delta Electronics (Americas) \\ shane.long@deltaww.com
}

\author{
Le Xie \\ Texas A\&M University \\ le.xie@tamu.edu *
}

\begin{abstract}
We propose a neural Lyapunov approach to assessing transient stability in power electronic-interfaced microgrid interconnections. The problem of transient stability assessment is cast as one of learning a neural network-structured Lyapunov function in the state space. Based on the function learned, a security region is estimated for monitoring the security of interconnected microgrids in real-time operation. The efficacy of the approach is tested and validated in a grid-connected microgrid and a three-microgrid interconnection. A comparison study suggests that the proposed method can achieve a less conservative characterization of the security region, as compared with a conventional approach [1].
\end{abstract}

\section{Introduction}

Microgrids provide promising solutions to enhancing the resiliency of distribution systems with increasing level of penetration of distributed energy resources (DERs) [2-5]. The flexibility of microgrids is enabled by their two operation modes: an islanded mode and a grid-connected mode [6]. In the grid-connected mode, one microgrid interconnects with the rest of the distribution system via the point of common coupling (PCC) [6] in order to achieve entire system-wide optimal operation. A microgrid is also capable of proactively transitioning to an islanded mode, once the main grid loses its desirable functions or severe faults in the microgrid compromise the security of the main grid $[3,4]$.

The control and management of grid-connected microgrids is typically hierarchically structured [68]. At the individual microgrid level, a microgrid central controller (MGCC) regulates the interconnected distributed generation units (DGUs) by tuning the setpoints of their local controllers [2, 6, 7]. At

The work of T. Huang and L. Xie is supported in part by NSF ECCS-1611301, 2038963, and DOE grant DE-EE0009031. the distribution-system level, a distribution system operator (DSO) coordinates interconnected microgrids by controlling microgrid interfaces, rather than directly regulating DGUs [2]. Voltage source inverters (VSI) generally serve as the interfaces of microgrids, where various control strategies, such as master/slave, current sharing, and frequency/angle droop methods $[2,7,8]$, can be implemented.

The rise of interest in deploying microgrids and interconnection of multiple microgrids provides many research challenges and opportunities in the design and operation of their energy management systems. One particular area of challenge is how to design safe and efficient transient stability assessment tools for future grid designers and operators. Transient stability assessment for microgrids essentially aims to answer the following question: Given an operating condition, to what extent of disturbances can the microgrids tolerate? A rigorous answer to such a question is much needed for both offline design and online operation of microgrids. For example, the stability assessment tool provides microgrids' designers with a criterion for optimal design scheme selection. Such an assessment tool also allows DSO to maintain greater situational awareness and helps them to decide if corrective actions need be taken after disturbances happen. The challenge of developing such an assessment tool lies in the fact that microgrids and their interconnections are nonlinear systems $[2,6]$ and the dynamics of such nonlinear systems under large disturbances need be analyzed.

In view of the above challenge, several techniques are proposed for stability assessment in both the microgrid level $[6,9-11]$ and the microgrid-interconnection level $[2,4,12-14]$. This paper focuses on the level of interconnected microgrids. Within such a research scope, one possible method is to leverage the energy function approach that is extensively studied in analysis of large-scale transmission systems. By assuming that the transmission lines are lossless, the energy function approach constructs a system behavior-summary function, namely, the energy 
function, in order to certify the stability of a equilibrium point. Reference [15] provides a thorough survey for the energy function approach in transmission systems. However, the qualitative difference between transmission systems and interconnected microgrids may prevent the energy approach developed for transmission systems to migrate to interconnected microgrids. For example, the lossless-line assumption is a valid assumption for transmission systems, but distribution lines in interconnected microgrids have a lower reactance to resistance ratio in comparison with transmission systems [6], rendering the lossless-line assumption invalid and, thereby, leading to the nonexistence of the energy function [1]. Without the lossless-line assumption, reference [2] decouples the slow and fast dynamics in the interconnected microgrid and analyzes them separately: The stability of slow dynamics is addressed by the linearization technique [16] widely applied in bulk transmission system applications [17-20]; and the transient stability of fast dynamics is assessed by linear matrix inequality (LMI). However, the proposed framework in [2] only provides a binary (yes/no) answer to transient stability of the interconnected microgrids. It is equally important to estimate the extent of disturbances that can be tolerated by interconnected microgrids.

This paper leverages the most recent advances in machine learning and control theory to provide rigorious and scalable assessment of transient stability in interconnected microgrids. A neural Lyapunov approach is proposed for assessing transient stability of a microgrid interconnection. The problem of transient stability assessment is cast as one of learning a neural network-structured Lyapunov function in the state space. Based on the function learned, a security region is estimated for monitoring the security of interconnected microgrids in real-time operation. The contribution of this paper is twofold: 1) It introduces a novel type of Lyapunov function that can rigorously establish asymptotic stability of interconnected microgrids and provide a security region that allows microgrids' designers/operators to estimate the extent of disturbances that a microgrid interconnection can tolerate; and 2) the proposed approach does not require a special form of interface dynamics of interconnected microgrids, allowing it to analyzing realistic microgrid interconnections.

The rest of this paper is organized as follows: Section 2 presents the mathematical description of interconnected microgrid dynamics; Section 3 elaborates procedures for learning a neural network-structured Lyapunov function and finding a security region by the Lyapunov function learned;
Section 4 tests and validates the proposed approach; and Section 5 concludes the paper and points out the future direction of this work.

\section{Dynamics of Networked Microgrids}

A future distribution system can be considered as an interconnection of $m$ PE-interfaced microgrids. For microgrid $i \in\{1,2, \ldots, m\}$, its PCC interface dynamics can be described by the following differential equations: $[2,4]$

$$
\begin{aligned}
T_{\mathrm{A} i} \dot{\delta}_{i} & =D_{\mathrm{A} i}\left(P_{i}^{*}-P_{i}\right)-\left(\delta_{i}-\delta_{i}^{*}\right) \\
T_{\mathrm{E} i} \dot{E}_{i} & =D_{\mathrm{E} i}\left(Q_{i}^{*}-Q_{i}\right)-\left(E_{i}-E_{i}^{*}\right),
\end{aligned}
$$

where state variables $\delta_{i}$ and $E_{i}$ are the voltage angle and voltage magnitude at the $i$-th PCC, respectively; algebraic variables $P_{i}$ and $Q_{i}$ are real and reactive power injections to the $i$-th PCC; setpoints $\delta_{i}^{*}, E_{i}^{*}, P_{i}^{*}$, and $Q_{i}^{*}$ are dispatched by a distribution system operator based on steady-state security/economic studies; control parameters $T_{\mathrm{A} i}$ and $T_{\mathrm{E} i}$ denote tracking time constants of voltage angle and magnitude at PCC $i$; and $D_{\mathrm{A} i}$ and $D_{\mathrm{E} i}$ are droop gains of voltage angle and magnitude at PCC $i[2,4]$. Note that equation (1) describes the interface dynamics with angle droop control. If the frequency droop control is deployed in microgrid interfaces, the swing-equation type of dynamics will replace (1).

Microgrid $i$ is interconnected with other microgrids through distribution lines which introduce the following algebraic constrains:

$$
\begin{aligned}
P_{i} & =E_{i}^{2} G_{i i}+\sum_{k \neq i} E_{i} E_{k} Y_{i k} \cos \left(\delta_{i}-\delta_{k}-\theta_{i k}\right),(2 \mathrm{a}) \\
Q_{i} & =-E_{i}^{2} B_{i i}+\sum_{k \neq i} E_{i} E_{k} Y_{i k} \sin \left(\delta_{i}-\delta_{k}-\theta_{i k}\right), \forall i,
\end{aligned}
$$

where $Y_{i k} \angle \theta_{i k}$ denotes the element at the $i$-th row and the $k$-th column of admittance matrix $Y ; G_{i i}$ and $B_{i i}$ are real and imaginary parts of $Y_{i i} \angle \theta_{i i}$, respectively. Suppose that the equilibrium point of the $m$-microgrid interconnection described by differential algebraic equations (DAEs) (1) and (2) is

$$
\mathbf{o}=\left[\delta_{1}^{*}, \delta_{2}^{*}, \ldots, \delta_{m}^{*}, E_{1}^{*}, E_{2}^{*}, \ldots, E_{m}^{*}\right]^{\top}
$$

where variables $\delta_{i}^{*}$ and $E_{i}^{*}$ for all $i \in 1,2, \ldots, m$ satisfy 


$$
\begin{aligned}
& P_{i}^{*}=E_{i}^{* 2} G_{i i}+\sum_{k \neq i} E_{i}^{*} E_{k}^{*} Y_{i k} \cos \left(\delta_{i}^{*}-\delta_{k}^{*}-\theta_{i k}\right) \\
& Q_{i}^{*}=-E_{i}^{* 2} B_{i i}+\sum_{k \neq i} E_{i}^{*} E_{k}^{*} Y_{i k} \sin \left(\delta_{i}^{*}-\delta_{k}^{*}-\theta_{i k}\right), \forall i .
\end{aligned}
$$

Next we modify (1) and (2) such that the equilibrium point of the modified DAEs is the origin of the state space. Define new state variables

$$
\begin{aligned}
\delta_{i}^{\prime} & =\delta_{i}-\delta_{i}^{*}, \\
E_{i}^{\prime} & =E_{i}-E_{i}^{*}, \forall i .
\end{aligned}
$$

With new state variables $\delta_{i}^{\prime}$ and $E_{i}^{\prime}$, the $i$-th PCC interface dynamics is characterized by differential equations

$$
\begin{aligned}
T_{\mathrm{A} i} \dot{\delta}_{i}^{\prime} & =D_{\mathrm{A} i}\left(P_{i}^{*}-P_{i}\right)-\delta_{i}^{\prime} \\
T_{\mathrm{E} i} \dot{E}_{i}^{\prime} & =D_{\mathrm{E} i}\left(Q_{i}^{*}-Q_{i}\right)-E_{i}^{\prime},
\end{aligned}
$$

with algebraic equations

$$
\begin{aligned}
& P_{i}=\left(E_{i}^{\prime}+E_{i}^{*}\right)^{2} G_{i i}+ \\
& \sum_{k \neq i}\left(E_{i}^{\prime}+E_{i}^{*}\right)\left(E_{k}^{\prime}+E_{k}^{*}\right) Y_{i k} \cos \left(\delta_{i k}^{\prime}+\delta_{i k}^{*}-\theta_{i k}\right), \\
& Q_{i}=-\left(E_{i}^{\prime}+E_{i}^{*}\right)^{2} B_{i i}+ \\
& \sum_{k \neq i}\left(E_{i}^{\prime}+E_{i}^{*}\right)\left(E_{k}^{\prime}+E_{k}^{*}\right) Y_{i k} \sin \left(\delta_{i k}^{\prime}+\delta_{i k}^{*}-\theta_{i k}\right), \forall i,
\end{aligned}
$$

where $\delta_{i k}^{\prime}=\delta_{i}^{\prime}-\delta_{k}^{\prime}$ and $\delta_{i k}^{*}=\delta_{i}^{*}-\delta_{k}^{*}$. The equilibrium point of the dynamic system described by (6) and (7) is the origin of the state space.

It is worth noting that if time constant $T_{\mathrm{E} i}$ is much greater than $T_{\mathrm{A} i}$, the evolution of $\delta_{i}^{\prime}$ is much faster than that of $E_{i}^{\prime}$. Therefore, when phase angles are the state variables of interest, $E_{i}^{\prime}$ in (6) can be assumed to be a constant [2], i.e., $E_{i}^{\prime}=0$. Such an assumption is called the time-scale separation assumption [4]. Under the time-scale separation assumption, the system equations (6) and (7) can be simplified to

$$
T_{\mathrm{A} i} \dot{\delta}_{i}^{\prime}=D_{\mathrm{A} i}\left(P_{i}^{*}-P_{i}\right)-\delta_{i}^{\prime}, \forall i,
$$

where

$$
P_{i}=E_{i}^{* 2} G_{i i}+\sum_{k \neq i} E_{i}^{*} E_{k}^{*} Y_{i k} \cos \left(\delta_{i k}^{\prime}+\delta_{i k}^{*}-\theta_{i k}\right) .
$$

The system equations (6) or (8) can be written in the following compact form:

$$
\dot{\mathbf{x}}=\mathbf{f}(\mathbf{x})
$$

where $\mathbf{f}(\cdot)$ corresponds to (8) if $T_{\mathrm{E} i} \gg T_{\mathrm{A} i}$, otherwise it associates with (6); and the state vector $\mathbf{x}$ is defined by

$\mathbf{x}:= \begin{cases}{\left[\delta_{1}^{\prime}, \delta_{2}^{\prime}, \ldots, \delta_{m}^{\prime}\right]^{\top}} & T_{\mathrm{E} i} \gg T_{\mathrm{A} i} \\ {\left[\delta_{1}^{\prime}, \delta_{2}^{\prime}, \ldots, \delta_{m}^{\prime}, E_{1}^{\prime}, E_{2}^{\prime}, \ldots, E_{m}^{\prime}\right]^{\top}} & \text { otherwise. }\end{cases}$

The equilibrium point $\mathbf{o}^{\prime}$ of (10) is an $m^{\prime}$-dimensional zero vector, i.e., $\mathbf{o}^{\prime}=\mathbf{0}_{m^{\prime}}$, where $m^{\prime}=m$ if $T_{\mathrm{E} i} \gg T_{\mathrm{A} i}$, otherwise $m^{\prime}=2 m$.

For the interconnected microgrids described by (10), it is highly desirable for microgrids' designers/operators to know: 1) whether the equilibrium point $\mathbf{o}^{\prime}$ is asymptotically stable; and 2) how much state deviation from $\mathbf{o}^{\prime}$ (caused by disturbances, say, line tripping) the interconnected microgrids can tolerate.

\section{Transient Stability Assessment}

This section presents a neural Lyapunov approach to transient stability assessment in the PE-interfaced microgrid interconnection described by (10). First, some background knowledge is presented in order to show that one possible way to answer the two questions raised at the end of Section 2 is to construct a Lyapunov function. Then the Lyapunov function is learned by minimizing the empirical Lyapunov risk [21] and augmenting training samples. Finally, a region of attraction is estimated based on the Lyapunov function learned.

\subsection{Background Definitions}

We consider the interconnected microgrids described by (10) with the equilibrium point $\mathbf{o}^{\prime}$ at the origin. A Lyapunov function [22] can be leveraged to establish the stability of the equilibrium point $\mathbf{o}^{\prime}$ and its definition is as follows:

Definition 1 If, in a ball $D_{R}:=\left\{\mathbf{x} \mid\|\mathbf{x}\|_{2}^{2} \leq R^{2}\right\}$, there exists a continuous differentiable scalar function $V$ such that

- $V$ is positive definite in $D_{R}$,

- $\dot{V}$ is negative definite in $D_{R}$

then the equilibrium point $\mathbf{o}^{\prime}$ is asymptotically stable, and the function $V$ is called a Lyapunov function.

In Definition 1, a positive/negative definite function in $D_{R}$ is defined as follows. A function $F(\mathbf{x})$ is a 
positive definite function in $D_{R}$, if $F(\mathbf{0})=0$ and $F(\mathbf{x})>0$ for all $\mathbf{x} \in D_{R} \backslash\{\mathbf{0}\}$. A function $F(\mathbf{x})$ is a negative definite function in $D_{R}$, if $-F(\mathbf{x})$ is a positive definite function in $D_{R} . \dot{V}(\mathbf{x})$ is the time derivative of $V(\mathbf{x})$ and it can be found by

$$
\dot{V}=\frac{d V(\mathbf{x})}{d t}=\frac{\partial V}{\partial \mathbf{x}} \mathbf{f}(\mathbf{x})
$$

Definition 1 essentially suggests that we can answer the first question raised at the end of Section 2 by searching for a legitimate Lyapunov function $V(\mathbf{x})$ : the asymptotic stability can be certified, if a Lyapunov function can be found.

We proceed to address the second question at the end of Section 2, viz., how much state deviation from $\mathbf{o}^{\prime}$ the interconnected microgrids can tolerate. Such a question boils down to finding a region in the state space such that the system trajectory initiating from the region never leaves the region and converges to the origin of the state space $[21,22]$. In this paper, such a region is called a security region $\mathcal{R}$, which is formally defined as follows:

Definition 2 A region $\mathcal{R} \subseteq \mathbb{R}^{m^{\prime}}$ is a security region if

$$
\mathbf{x}(0) \in \mathcal{R} \Longrightarrow \mathbf{x}(\infty)=\mathbf{0}_{m^{\prime}} \wedge \forall t>0(\mathbf{x}(t) \in \mathcal{R}) .
$$

In Definition 2, $\mathbf{0}_{m^{\prime}}$ is an $m^{\prime}$-dimension zero vector; and " $\wedge$ " means "and." Based on Definition 2, a security region is a subset of the domain of attraction [22]. If a Lyapunov function $V(\mathbf{x})$ is available, one estimation for the security region $\mathcal{R}$ is

$$
\mathcal{R}_{c}=\left\{\mathbf{x} \in D_{R} \mid c \in \mathbb{R}_{+}, V(\mathbf{x})<c\right\} .
$$

The security region estimation $\mathcal{R}_{c}$ can be leveraged for online stability assessment for interconnected microgrids: if the state deviation from the pre-dispatched equilibrium $\mathbf{o}^{\prime}$ falls within $\mathcal{R}_{c}$, the system is guaranteed to be secure, as state deviations never leave $\mathcal{R}_{c}$ and tend to zeros as time goes to infinity.

Definition 1 and equation (13) can be used to 1) certify the asymptotic stability and 2) estimate a security region around an equilibrium point dispatched by DSO. However, the prerequisite to achieve the above two goal is the availability of a Lyapunov function. In what follows we present how to find such a function using neural network.

\subsection{Neural Network-structured Lyapunov Function}

We assume that the Lyapunov function $V(\mathbf{x})$ has a neural-network structure, as such a structure enables us to approximate a wide class of functions. The input of the neural network is state vector $\mathbf{x}$, and the output is the value of $V$ evaluated at $\mathbf{x}$. The fundamental computing units of the neural-network are neurons which is a multiple-input-single-output system [23-25]. Suppose that the input of the neuron $i$ is $\mathbf{x}_{0}$. Neuron $i$ conducts a two-step computing procedure. First, neuron $i$ takes linear combination of its input and obtain an intermediate variable $d_{i}$, i.e.,

$$
d_{i}=\mathbf{w}_{i} \mathbf{x}_{0}+b_{i}
$$

where $\mathbf{w}_{i} \in \mathbb{R}^{1 \times\left|\mathbf{x}_{0}\right|}$ collects weighting coefficients; and $b_{i} \in \mathbb{R}$ is a bias coefficient. Second, neuron $i$ evaluates a nonlinear function (a.k.a. the activation function) at $\mathbf{x}_{0}$ and returns the function value as its output $y_{0}$. In this paper, the hyperbolic tangent function is chosen to be the activation function, i.e.,

$$
y_{i}=\tanh \left(d_{i}\right) .
$$

The neurons are interconnected in a layered architecture and constitute a neural network. A neural network comprises an input layer, at least one hidden layer, and an output layers. This paper uses a neural network with one hidden layer to approximate the Lyapunov function $V(\mathbf{x})$.

We proceed to analyze the neurons in the hidden layer. Let $\mathbf{d}^{[1]}$ collect all intermediate variables of neurons in the hidden layer, i.e.,

$$
\mathbf{d}^{[1]}:=\left[d_{1}^{[1]}, d_{2}^{[1]}, \ldots, d_{n_{\mathrm{H}}}^{[1]}\right]^{\top}
$$

where $d_{i}^{[1]}$ denotes the intermediate variable of the $i$-th neuron in the first layer, viz., the only hidden layer; and $n_{\mathrm{H}}$ is the number of neurons in the hidden layer. Similarly, define $\mathbf{y}^{[1]}$ by

$$
\mathbf{y}^{[1]}:=\left[y_{1}^{[1]}, y_{2}^{[1]}, \ldots, y_{n_{\mathrm{H}}}^{[1]}\right]^{\top}
$$

where $y_{i}^{[1]}$ denotes the output of the $i$-th neuron in the first layer. The two-step procedure of neurons in the hidden layer leads to

$$
\begin{aligned}
& \mathbf{d}^{[1]}=W^{[1]} \mathbf{x}+\mathbf{b}^{[1]} \\
& \mathbf{y}^{[1]}=\tanh \left(\mathbf{d}^{[1]}\right)
\end{aligned}
$$

where $W^{[1]}:=\left[w_{j, k}^{[1]}\right]$ collects the weighting coefficients of the neurons in the hidden layer, whence its $(j, k)$-th entry is the weighting coefficient for the $k$-th input of the $j$-th neuron; column vector $\mathbf{b}^{[1]} \in \mathbb{R}^{n_{\mathrm{H}}}$ collects bias coefficients; and tanh denotes the element-wised hyperbolic tangent function. 
Following the similar notation system, for the output layer, we have

$$
\begin{aligned}
& d^{[2]}=W^{[2]} \mathbf{y}^{[1]}+b^{[2]} \\
& y^{[2]}=\tanh \left(d^{[2]}\right)
\end{aligned}
$$

where $d^{[2]}$ and $b^{[2]}$ are the intermediate variable and bias coefficient vector of the neuron at the second (output) layer, respectively; $W^{[2]} \in \mathbb{R}^{1 \times n_{\mathrm{H}}}$ collects weighting coefficients of the neuron at the output layer; and $y^{[2]} \in$ $\mathbb{R}$ is the output of the neural network.

Let a vector $\boldsymbol{\alpha}$ collect all unknown parameters in $W^{[1]}, W^{[2]}, \mathbf{b}^{[1]}$, and $b^{[2]}$. One can randomly draw $n$ state vectors $\mathbf{x}_{1}, \mathbf{x}_{2}, \ldots, \mathbf{x}_{n}$ from the state space and evaluate output of the neural network with parameters $\boldsymbol{\alpha}$. These state vectors can be considered as training examples for the neural network. A key question is how to tune parameters $\boldsymbol{\alpha}$ such that the neural network represented by (18) and (19) behaves like a Lyapunov function. Such a question is addressed by introducing a cost function in the following sections.

\subsection{Empirical Lyapunov Risk}

In order to enable the neural network described by (18) and (19) to behave like a Lyapunov function, the following cost function, i.e., empirical Lyapunov risk, is introduced to update parameters $\boldsymbol{\alpha}$ :

$$
\begin{array}{r}
L_{n, \rho}(\boldsymbol{\alpha})=\frac{1}{n} \sum_{k=1}^{n} \max \left(0,-V_{\boldsymbol{\alpha}}\left(\mathbf{x}_{k}\right)\right)+ \\
\frac{1}{n} \sum_{k=1}^{n} \max \left(0, \dot{V}_{\boldsymbol{\alpha}}\left(\mathbf{x}_{k}\right)\right)
\end{array}
$$

where $n$ is the number of training examples; and $\rho$ is the probability distribution according to which the training examples are drawn. If $V_{\boldsymbol{\alpha}}$ is not a legitimate Lyapunov function, positive penalties can be incurred in the the cost function $L_{n, \rho}(\boldsymbol{\alpha})$. The parameters $\boldsymbol{\alpha}^{*}$ of a legitimate Lyapunov function can minimize the cost function $L_{n, \rho}(\boldsymbol{\alpha})$. The cost function (20) is called the Lyapunov risk in [21]. In practice, the "max" function in (20) can be replaced by the Rectified Linear Unit (ReLU) which is defined by

$$
\operatorname{ReLU}(z)= \begin{cases}z & z \geq 0 \\ 0 & z<0 .\end{cases}
$$

Equation (20) is equivalent to

$L_{n, \rho}(\boldsymbol{\alpha})=\frac{1}{n} \sum_{k=1}^{n}\left(\operatorname{ReLU}\left(-V_{\boldsymbol{\alpha}}\left(\mathbf{x}_{k}\right)\right)+\operatorname{ReLU}\left(\dot{V}_{\boldsymbol{\alpha}}\left(\mathbf{x}_{k}\right)\right)\right)$.

Besides, $\dot{V}_{\boldsymbol{\alpha}}$ can be evaluated by (12), viz.,

$$
\dot{V}_{\boldsymbol{\alpha}}=\frac{\partial V_{\boldsymbol{\alpha}}}{\partial \mathbf{x}} \mathbf{f}(\mathbf{x}) .
$$

Since the Lyapunov function candidate $V_{\boldsymbol{\alpha}}\left(=y^{[2]}\right)$ has a neural-network structure, $\left(\frac{\partial V_{\alpha}}{\partial \mathbf{x}}\right)$ can be expressed in terms of the parameters in set $\boldsymbol{\alpha}$ according to the chain rule:

$$
\frac{\partial V_{\boldsymbol{\alpha}}}{\partial \mathbf{x}}=\frac{\partial V_{\boldsymbol{\alpha}}}{\partial d^{[2]}} \frac{\partial d^{[2]}}{\partial \mathbf{y}^{[1]}} \frac{\partial \mathbf{y}^{[1]}}{\partial \mathbf{d}^{[1]}} \frac{\partial \mathbf{d}^{[1]}}{\partial \mathbf{x}} .
$$

In (24),

$$
\begin{aligned}
& \frac{\partial V_{\boldsymbol{\alpha}}}{\partial d^{[2]}}=1-V_{\boldsymbol{\alpha}}^{2}, \quad \frac{\partial d^{[2]}}{\partial \mathbf{y}^{[1]}}=W^{[2]}, \quad \frac{\partial \mathbf{d}^{[1]}}{\partial \mathbf{x}}=W^{[1]}, \\
& \frac{\partial \mathbf{y}^{[1]}}{\partial \mathbf{d}^{[1]}}=\operatorname{diag}\left(1-\tanh ^{2}\left(d_{1}^{[1]}\right), \ldots, 1-\tanh ^{2}\left(d_{n_{\mathrm{H}}}^{[1]}\right)\right),
\end{aligned}
$$

where

$$
d_{k}^{[1]}=W_{k,:}^{[1]} \mathbf{x}+b_{k}^{[1]}, \quad k=1,2, \ldots, n_{\mathrm{H}} .
$$

whence $W_{k,:}^{[1]}$ denotes the $k$-th row of matrix $W^{[1]}$; and $b_{k}^{[1]}$ is the $k$-th entry of $\mathbf{b}^{[1]}$.

Suppose that we have a training set $\mathcal{X}$ with $n$ i.i.d. examples randomly drawn from the state space, i.e.,

$$
\mathcal{X}:=\left\{\mathbf{x}_{1}, \mathbf{x}_{2}, \ldots, \mathbf{x}_{k}, \ldots, \mathbf{x}_{n}\right\}
$$

where $\mathbf{x}_{k} \sim \rho$ for $k=1,2, \ldots, n$. Based on the training set $\mathcal{X}$, one can find the coefficients of a neural network-structured Lyapunov function candidate by minimizing the Lyapunov risk, i.e.,

$$
\min _{\boldsymbol{\alpha}} L_{n, \rho}(\boldsymbol{\alpha}) .
$$

The optimization problem (27) can be solved by the stochastic gradient decent method. The pseudo-code of the method is presented in Algorithm 1 where $\boldsymbol{\alpha}_{0}$ is the initial guess of the parameters; and $\eta$ is the learning rate specified by users.

\subsection{Training Set Augment}

The Lyapunov function candidate with parameters $\boldsymbol{\alpha}$ that is returned by Algorithm 1 may not be a legitimate 


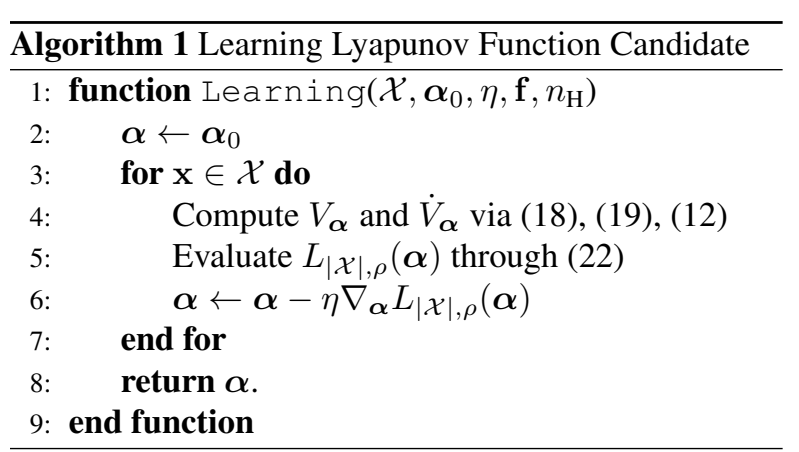

Lyapunov function. The reason is that the function with $\boldsymbol{\alpha}$ might violate the two conditions in Definition 1 when it is evaluated at the training examples that are not in $\mathcal{X}$. Therefore, it is necessary to find counterexamples that lead the candidate function $V_{\boldsymbol{\alpha}^{*}}$ to violate the two conditions in Definition 1 and add the counterexamples to the training set $\mathcal{X}$.

Recent advances in satisfiability modulo theories (SMT) can be leveraged to find the counterexamples. The SMT solver finds the counterexamples by checking the satisfiability of the following condition:

$$
\left(\|\mathbf{x}\|_{2}^{2} \geq r^{2}\right) \wedge\left(V_{\boldsymbol{\alpha}}(\mathbf{x}) \leq 0 \vee \dot{V}_{\boldsymbol{\alpha}}(\mathbf{x}) \geq 0\right)
$$

where $\mathbf{x} \in D_{R} ; r \ll \min \left(1,\|x\|_{2}\right)$ and " $\vee$ " denotes "or". The condition $\|\mathbf{x}\|_{2}^{2} \geq r^{2}$ is added for avoiding numerical issues [21]. The SMT solver, such as dReal [26], can return a set $\mathcal{S}$ that comprises some x satisfying condition (28). If no $\mathbf{x}$ can be found in $D_{R}, \mathcal{S}$ is an empty set. Such a procedure is presented in Algorithm 2. Algorithm 3 shows the overall procedure to find a legitimate Lyapunov function.

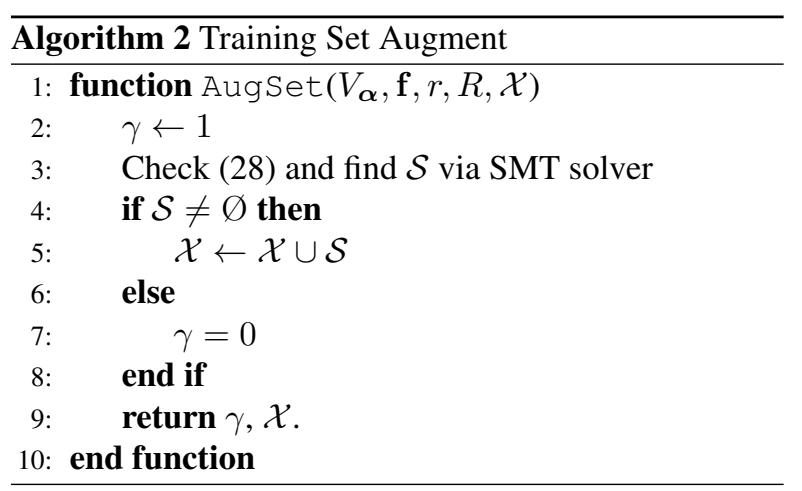

\subsection{Security Region Estimation}

Given the neural network-structured Lyapunov function $V_{\boldsymbol{\alpha}^{*}}$ learned from the state space, a security

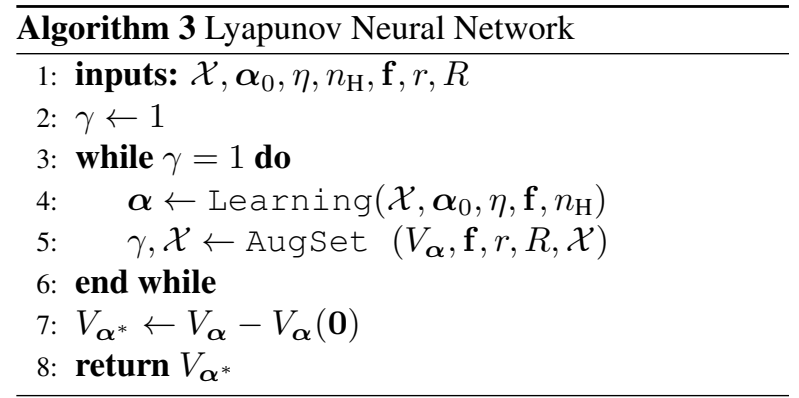

region can be estimated by (13). As $c$ in (13) increases, the estimated security region is enlarged. However, by (13), the security region should be a subset of the valid region $D_{R}$, indicating that $c$ cannot be too large. Denote by $c^{*}$ the optimal $c$ that maximizes the security region estimation $\mathcal{R}_{c}$. One observation [27] is that

$$
c^{*}=\min _{\mathbf{x} \in \partial D_{R}} V_{\boldsymbol{\alpha}^{*}}(\mathbf{x})
$$

where $\partial D_{R}:=\left\{\mathbf{x} \mid\|\mathbf{x}\|_{2}^{2}=R^{2}\right\}$.

We use the Lagrange multiplier method to solve (29). The Lagrangian $L(\mathbf{x}, v)$ associated with (29) is

$$
L(\mathbf{x}, v)=V_{\boldsymbol{\alpha}^{*}}(\mathbf{x})+v\left(\|\mathbf{x}\|_{2}^{2}-R^{2}\right)
$$

where $v \in \mathbb{R}$ is a Lagrangian multiplier. Critical points for (29) can be found by solving the following nonlinear equations for $\mathbf{x}$ and $v$ :

$$
\begin{aligned}
& \frac{\partial L(\mathbf{x}, v)}{\partial \mathbf{x}}=0 \\
& \|\mathbf{x}\|_{2}^{2}=R^{2}
\end{aligned}
$$

Suppose that there are $p$ critical points which are collected by $\mathcal{H}:=\left\{\hat{\mathbf{x}}_{1}, \hat{\mathbf{x}}_{2}, \ldots, \hat{\mathbf{x}}_{p}\right\}$. The local maxima and minima occur at these critical points in $\mathcal{H}$. Furthermore, the global minima can be obtained by

$$
c^{*}=\min _{\mathbf{x} \in \mathcal{H}} V_{\boldsymbol{\alpha}^{*}}(\mathbf{x})
$$

The security region that is used for online transient stability assessment in a $m$-microgrid interconnection is

$$
\mathcal{R}_{c^{*}}=\left\{\mathbf{x} \mid \mathbf{x} \in D_{R}, V(\mathbf{x})<c^{*}\right\} .
$$

\section{Case Study}

This section focuses on testing and validating the neural Lyapunov approach. For the convenience of visualization, we start from a single grid-connected microgrid with two state variables. Then, the 
proposed approach is validated in three PE-interfaced interconnected microgrids. These test cases are implemented in a Python environment and the algorithms are built upon some open-source packages. Algorithm 1 is implemented based on PyTorch. The SMT solver in Algorithm 2 is dReal [26]. Besides, nonlinear equations (31) are solved by SciPy.

\subsection{Single Grid-connected Microgrid}

In this subsection, the neural Lyapunov approach is tested and validated in a grid-connected microgrid (Figure 1). A microgrid with PCC 1 is connected to the rest of the grid via a distribution line whose impedance is $1.20+j 1.10$ p.u. The pre-dispatched setpoints for microgrid 1 are $P_{1}^{*}=0.3$ p.u., $Q_{1}^{*}=-0.16$ p.u., $E_{1}^{*}=1.05$ p.u., and $\delta_{1}^{*}=\angle 30^{\circ}$. The control parameters of microgrid 1 are $T_{\mathrm{A} 1}=1.2, D_{\mathrm{A} 1}=0.2, T_{\mathrm{V} 1}=12$, $D_{\mathrm{V} 1}=0.2$. The terminal behavior of the rest of the grid is modeled as a constant voltage source and its terminal voltage is 1.00 p.u. The time-scale separation is not assumed, so we have two state variables $\delta_{1}^{\prime}$ and $E_{1}^{\prime}$ which are the deviations of $\delta_{1}$ and $E_{1}$ from their equilibrium points. Note that the transient stability of such a system cannot be assessed by the LMI-based framework in [2], as it requires a special form of dynamics.

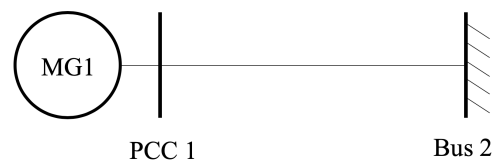

Figure 1. A grid-connected microgrid

Algorithm 3 is used to learn a Lyapunov function $V_{\boldsymbol{\alpha}^{*}}$. In Algorithm 3, $\eta=0.01, n_{\mathrm{H}}=6, r=0.5$, and $R=1$. There are 25 trainable parameters. After 370 iterations which take 18.69 seconds, Algorithm 3 outputs a Lyapunov function. Figure 2 visualizes the Lyapunov function learned in the $\delta_{1}^{\prime}-E_{1}^{\prime}-V$ space. As it can be observed in Figure 2, the function is positive-definite within the valid region $D_{R}$ (inside the red-dash circle). Figure 3 shows the visualization of $\dot{V}_{\boldsymbol{\alpha}^{*}}$, suggesting that $\dot{V}_{\boldsymbol{\alpha}^{*}}$ is a negative-definite function within the valid region $D_{R}$. Figures 2 and 3 indicate that the function learned indeed behaves like a Lyapunov function.

Given the Lyapunov function learned $\dot{V}_{\boldsymbol{\alpha}^{*}}$ and its valid region $D_{R}$, by (32), we have $c^{*}=0.9205$ at $\delta_{1}^{\prime}=-0.2953$ and $E_{1}^{\prime}=0.9554$. Through (33), we have a security region which is visualized in Figure 4. In Figure 4, what is inside the red-solid circle is the security region. Besides, the boundary of the security

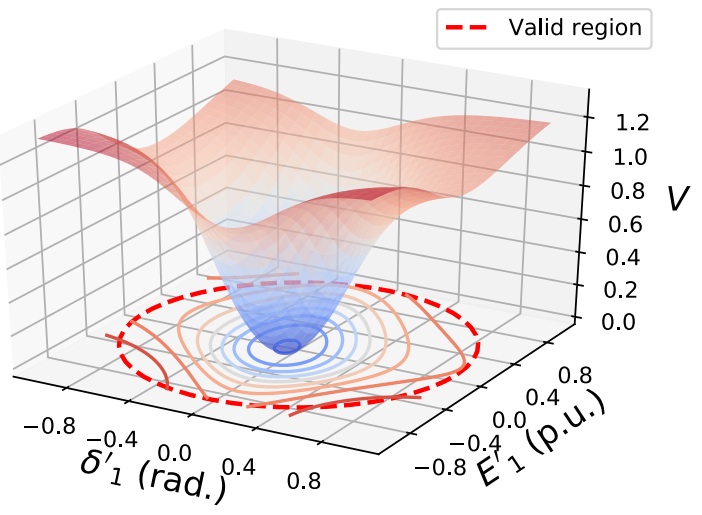

Figure 2. Lyapunov function for the single grid-connected microgrid

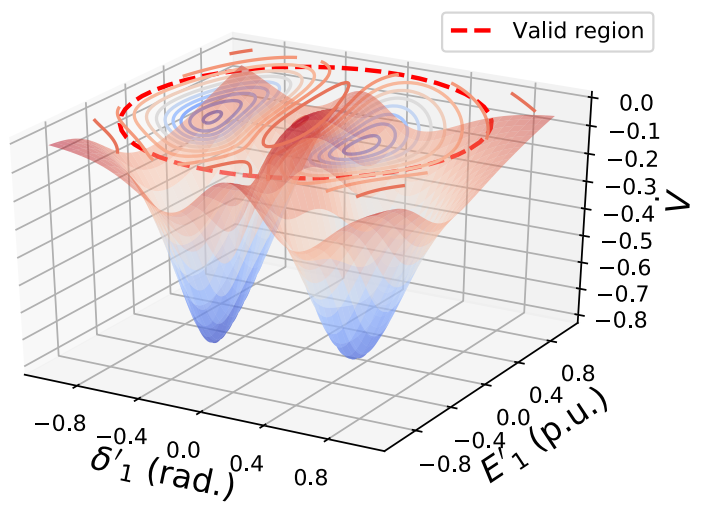

Figure 3. Lie derivative of Lyapunov function for the single grid-connected microgrid

region (the red-solid circle) intersects with the boundary of the valid region $D_{R}$ (the red-dash circle) at point $(-0.2953,0.9554)$, which is consistent with the result obtained from (32).

We proceed to compare the proposed neural-network approach (NN) with the standard approach (std.) reported in [1]. The comparison result is summarized in Figure 4 where the region inside the blue-solid circle is the security region estimated from the standard approach while the blue-dashed circle is the valid region boundary of the Lyapunov function from the standard approach. It can be observed that the security region estimated from the proposed approach (the region inside the red-solid circle) is larger than the one from the standard approach. This suggests the proposed method is less conservative than the standard approach.

\subsection{Three-microgrid Interconnection}

This subsection leverages a three-microgrid interconnection (Figure 5) to test and validate the 


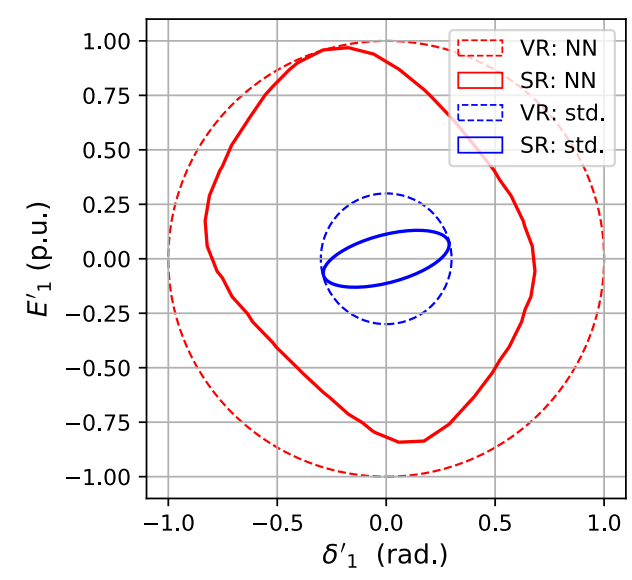

Figure 4. Visualization of estimated security region (SR) and valid region (VR)

proposed approach. In Figure 5, each microgrid is connected to the rest of the grid through distribution lines whose parameters are listed in Table 1 . We assume that there is clear time-scale separation and the voltage angle deviations, i.e., $\delta_{i}^{\prime}$ for $i=1,2,3$, are state variables of interest. The control parameters for each microgrid are listed in Table 2. Besides, the pre-designed setpoints are shown in Table 3

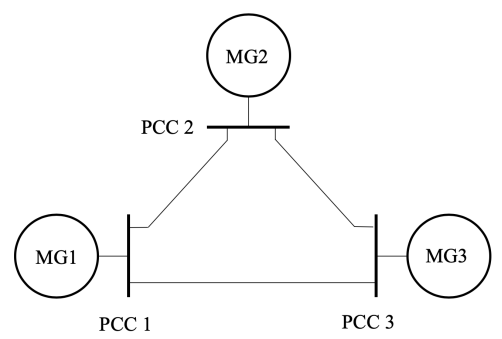

Figure 5. Topology of a three-microgrid interconnection

Table 1. Distribution line parameters of the three-microgrid interconnection

\begin{tabular}{c|c|c}
\hline Branch & $R$ (p.u.) & $X$ (p.u.) \\
\hline $1-2$ & 1.2030 & 1.1034 \\
\hline $1-3$ & 1.5042 & 1.3554 \\
\hline $2-3$ & 1.4680 & 1.1550 \\
\hline
\end{tabular}

A neural network-structured Lyapunov function is learned through Algorithm 3 where $\eta=0.01, n_{\mathrm{H}}=$ $6, r=0.5$, and $R=1$. There are 31 trainable parameters. After 630 iterations which take 169.13 seconds, Algorithm 3 returns a Lyapunov function. Figures 6 and 7 visualize the Lyapunov function learned and its time derivative in three-dimension space, where
Table 2. Control parameters of the three-microgrid interconnection

\begin{tabular}{c|c|c|c}
\hline & MG 1 & MG 2 & MG 3 \\
\hline$T_{\mathrm{A} i}$ & 1.2 & 1.0 & 0.8 \\
\hline$D_{\mathrm{A} i}$ & 0.2 & 0.2 & 0.2 \\
\hline$T_{\mathrm{V} i}$ & 12 & 10 & 16 \\
\hline$D_{\mathrm{V} i}$ & 0.2 & 0.2 & 0.2 \\
\hline
\end{tabular}

Table 3. Pre-dispatched Setpoints of the three-microgrid interconnection

\begin{tabular}{c|c|c|c}
\hline & MG 1 & MG 2 & MG 3 \\
\hline$\delta_{i}^{*}$ & 0 & $55.67^{\circ}$ & $-45.37^{\circ}$ \\
\hline$E_{i}^{*}$ (p.u.) & 1 & 1.05 & 0.95 \\
\hline$P^{*}$ (p.u.) & 0.1706 & 1.4578 & -0.0013 \\
\hline$Q^{*}$ (p.u.) & 0.4222 & -0.1639 & 1.0997 \\
\hline
\end{tabular}

state variable $\delta_{3}^{\prime}$ is set to zero for the convenience of visualization. Some supplementary figures are provided in Appendix A. As it can be observed in Figures 6 and 7, the function learned behaves like a Lyapunov function within the valid region $D_{R}$ (inside the red-dash circle), as the function learned is positive except around the origin, and its time derivative is negative except around the origin.

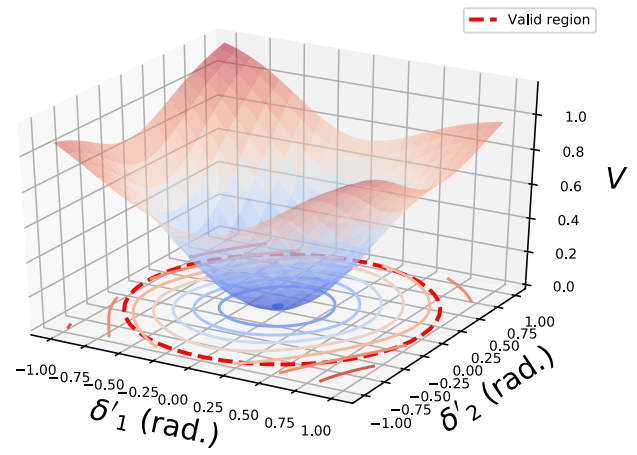

Figure 6. Visualization of the Lyapunov function in the $\delta_{1}^{\prime}-\delta_{2}^{\prime}-V$ space.

We proceed to estimate the security region based on the learned Lyapunov function $V_{\boldsymbol{\alpha}^{*}}$. By (32), we have $c^{*}=0.7037$. Therefore, the estimated security region is

$$
\mathcal{R}_{0.7037}=\left\{\mathbf{x} \mid\|\mathbf{x}\|_{2}^{2} \leq 1, V_{\boldsymbol{\alpha}^{*}}(\mathbf{x})<0.7037\right\} .
$$

Next we show how to utilize such a region to monitor the security of the interconnected microgrids in real-time operation. Suppose that due to some disturbances, the system state deviation becomes $\mathbf{x}(0)=[0.9150,0.1886,-0.1980]$, i.e., $\delta_{1}^{\prime}(0)=0.9150$ rad., $\delta_{2}^{\prime}(0)=0.1886$ rad., and $\delta_{3}^{\prime}(0)=-0.1980$ rad. Such initial states fall within the security region $\mathcal{R}_{0.7037}$, suggesting that the system trajectory will 


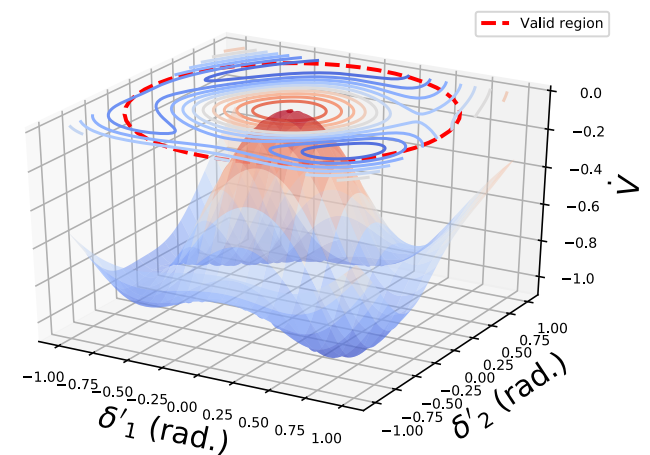

Figure 7. Visualization of the time derivative of Lyapunov function in the $\delta_{1}^{\prime}-\delta_{2}^{\prime}-\dot{V}$ space

stay within the security region and converge to the pre-dispatched equilibrium point. Such an assessment does not require any time-domain simulation and it is almost instantaneous.

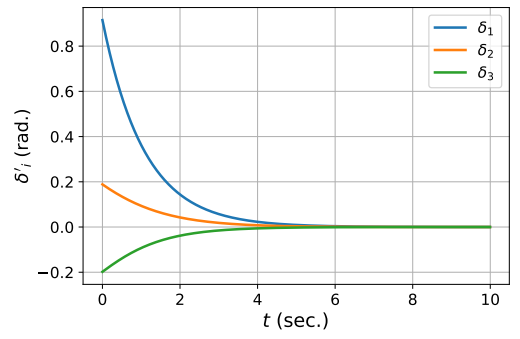

Figure 8. System response in time domain given the initial condition

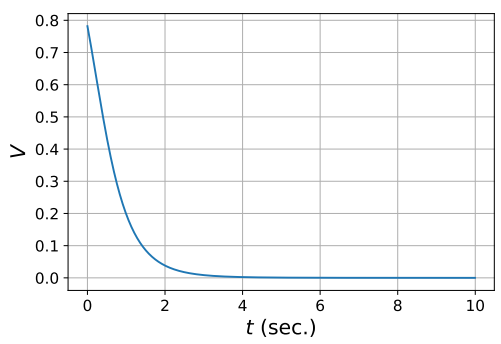

Figure 9. Evolution of Lyapunov function along a system trajectory

Figure 8 shows the time-domain simulation result with the initial condition $\mathbf{x}(0)$. As it can be observed, the states indeed converge to the pre-designed equilibrium point which is the origin of the state space. The result of the proposed approach matches the simulation result. Besides, with the initial condition $\mathbf{x}(0)$, the learned Lyapunov function can be evaluated in each time instant. The time-domain evolution of the Lyapunov function learned is presented in Figure 9, showing that the function learned is positive and it strictly decreases before the system reaches the equilibrium point.

\section{Conclusion}

In this paper, we propose a neural Lyapunov approach to assessing transient stability in power electronic-interfaced microgrid interconnections. Transient stability assessment is formulated as a problem of learning a neural network-structured Lyapunov function in the state space. Based on the function learned, a security region is estimated for monitoring the security of interconnected microgrids in real-time operation. The effectiveness of the approach is tested and validated in a grid-connected microgrid and a three-microgrid interconnection. Future work will investigate the scalability and conservativeness of this approach in larger systems.

\section{A. Appendix: Supplementary Figures}

Figures 10, 11, 12, and 13 visualize the learned Lyapunov function and its time derivative in three-dimension space. In Figures 10 and 13, $\delta_{2}^{\prime}$ is set to zero, while $\delta_{1}^{\prime}$ is set to zero in Figures 12 and 13 .

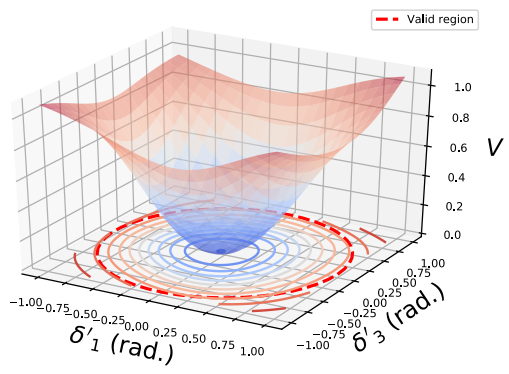

Figure 10. Visualization of the Lyapunov function in the $\delta_{1}^{\prime}-\delta_{3}^{\prime}-V$ space.

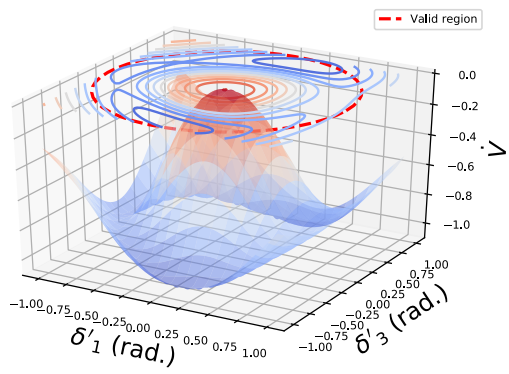

Figure 11. Visualization of the time derivative of Lyapunov function in the $\delta_{1}^{\prime}-\delta_{3}^{\prime}-\dot{V}$ space 


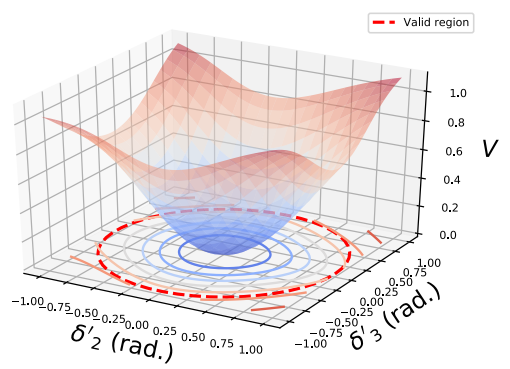

Figure 12. Visualization of the Lyapunov function in the $\delta_{2}^{\prime}-\delta_{3}^{\prime}-V$ space.

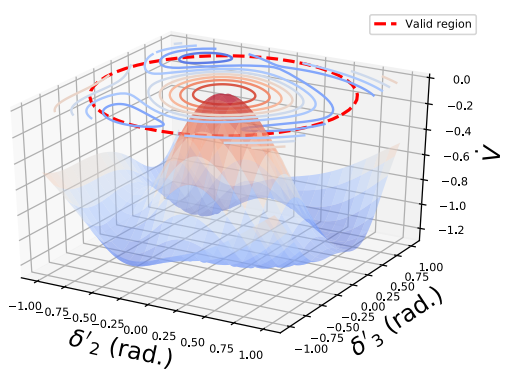

Figure 13. Visualization of the time derivative of Lyapunov function in the $\delta_{2}^{\prime}-\delta_{3}^{\prime}-\dot{V}$ space

\section{References}

[1] H. . Chiang, "Study of the existence of energy functions for power systems with losses," IEEE Transactions on Circuits and Systems, vol. 36, no. 11, 1989.

[2] Y. Zhang and L. Xie, "A transient stability assessment framework in power electronic-interfaced distribution systems," IEEE Transactions on Power Systems, vol. 31, no. 6, 2016.

[3] Z. Li et al., "Networked microgrids for enhancing the power system resilience," Proceedings of the IEEE, vol. 105, no. 7, pp. 1289-1310, 2017.

[4] T. Huang, H. Sun, K. J. Kim, D. Nikovski, and L. Xie, "A holistic framework for parameter coordination of interconnected microgrids against disasters," in IEEE PES GM, 2020.

[5] T. Huang, B. Wang, J. Ramos-Ruiz, P. Enjeti, P. Kumar, and L. Xie, "Detection of cyber attacks in renewable-rich microgrids using dynamic watermarking," in IEEE PES General Meeting, 2020.

[6] M. Farrokhabadi et al., "Microgrid stability definitions, analysis, and examples," IEEE Transactions on Power Systems, vol. 35, no. 1, pp. 13-29, 2020.

[7] J. M. Guerrero et al., "Hierarchical control of droop-controlled ac and dc microgrids - a general approach toward standardization," IEEE Transactions on Industrial Electronics, vol. 58, no. 1, pp. 158-172, 2011.

[8] R. Majumder et al., "Improvement of stability and load sharing in an autonomous microgrid using supplementary droop control loop," IEEE Transactions on Power Systems, vol. 25, no. 2, pp. 796-808, 2010.

[9] M. Kabalan et al., "Large signal lyapunov-based stability studies in microgrids: A review," IEEE Transactions on Smart Grid, vol. 8, no. 5, pp. 2287-2295, 2017.
[10] M. Ilić, R. Jaddivada, and X. Miao, "Modeling and analysis methods for assessing stability of microgrids," IFAC-PapersOnLine, vol. 50, no. 1, 2017.

[11] N. Pogaku, M. Prodanovic, and T. C. Green, "Modeling, analysis and testing of autonomous operation of an inverter-based microgrid," IEEE Transactions on Power Electronics, vol. 22, no. 2, pp. 613-625, 2007.

[12] I. P. Nikolakakos et al., "Stability evaluation of interconnected multi-inverter microgrids through critical clusters," IEEE Transactions on Power Systems, vol. 31, no. 4, pp. 3060-3072, 2016.

[13] Y. Zhang, L. Xie, and Q. Ding, "Interactive control of coupled microgrids for guaranteed system-wide small signal stability," IEEE Transactions on Smart Grid, vol. 7, no. 2, pp. 1088-1096, 2016.

[14] Y. Zhang and L. Xie, "Online dynamic security assessment of microgrid interconnections in smart distribution systems," IEEE Transactions on Power Systems, vol. 30, no. 6, pp. 3246-3254, 2015.

[15] Hsiao-Dong Chang, Chia-Chi Chu, and G. Cauley, "Direct stability analysis of electric power systems using energy functions: theory, applications, and perspective," Proceedings of the IEEE, vol. 83, no. 11, 1995.

[16] P. W. Sauer and M. A. Pai, Power system dynamics and stability, vol. 101. Prentice hall Upper Saddle River, NJ, 1998.

[17] T. Huang, M. Wu, and L. Xie, "Prioritization of pmu location and signal selection for monitoring critical power system oscillations," IEEE Transactions on Power Systems, vol. 33, no. 4, pp. 3919-3929, 2018.

[18] T. Huang, N. M. Freris, P. R. Kumar, and L. Xie, "Localization of forced oscillations in the power grid under resonance conditions," in 2018 52nd Annual Conference on Information Sciences and Systems (CISS), 2018.

[19] T. Huang, N. M. Freris, P. R. Kumar, and L. Xie, "A synchrophasor data-driven method for forced oscillation localization under resonance conditions," IEEE Transactions on Power Systems, 2020.

[20] T. Huang, B. Satchidanandan, P. R. Kumar, and L. Xie, "An online detection framework for cyber attacks on automatic generation control," IEEE Transactions on Power Systems, vol. 33, no. 6, 2018.

[21] Y.-C. Chang, N. Roohi, and S. Gao, "Neural lyapunov control," in Advances in Neural Information Processing Systems 32, Curran Associates, Inc., 2019.

[22] J.-J. E. Slotine, W. Li, et al., Applied nonlinear control, vol. 199. Prentice hall Englewood Cliffs, NJ, 1991.

[23] P. H. Winston, "Artificial intelligence, 1992."

[24] A. Ng, "Cs229 lecture notes," CS229 Lecture notes, 2000 .

[25] M. S. Modarresi, T. Huang, H. Ming, and L. Xie, "Robust phase detection in distribution systems," in 2017 IEEE Texas Power and Energy Conference (TPEC), pp. 1-5, 2017.

[26] S. Gao, J. Avigad, and E. Clarke, "Delta-complete decision procedures for satisfiability over the reals."

[27] T. L. Vu and K. Turitsyn, "Lyapunov functions family approach to transient stability assessment," IEEE Transactions on Power Systems, vol. 31, no. 2, 2016. 